\title{
Technologies in DNA diagnostics on the horizon
}

To be cost-effective and widely accepted, the next generation of instruments and devices for DNA diagnostics must be more compact, less labour-intensive and with a high throughput. This trend towards miniaturization using, for example, DNA chipbased formats, provides a concomitant reduction in reagent and equipment costs but it poses new challenges in areas of robotics, microfluidics, micromachining, microchemistry, nucleic acid chemistry and surface chemistry. What follows is a closer look at a few of the lucky winners. It is not meant to be exhaustive.

The Affymetrix-Molecular Dynamics joint venture plans to develop a hand-held diagnostic unit that can be used in a doctor's office for on-the-spot tests. It is envisioned that the miniature integrated nucleic acid diagnostic, or MIND, device will perform a number of integrated functions - from introduction of the sample of whole blood, extraction of the DNA, to amplification of the target DNA and analysis either by capillary-array electrophoresis or DNA probe-array hybridization.

The DNA probe-based system builds on Affymetrix's VLSIPS (very-large-scale immobilized polymer synthesis) technology, which uses a clever combination of photolithographic techniques common to the semiconductor industry and solid-phase chemical synthesis. The process allows for the rapid and automated synthesis of miniaturized, high-density arrays of complex DNA probes in specific locations on the surface of a tiny silicon microchip. Molecular Dynamics will develop capillary array electrophoresis, a rapid method for separating and sizing DNA fragments, into a robust and reusable system. Early prototypes of the integrated device will be used to detect mutations that confer drug resistance in AIDS patients in the hope that it will assist doctors when prescribing antivirals, as well as tests for fragile $X$ syndrome and Duchenne mus-

Most of the awards were made to single companies and limited to $\$ 2$ million or less over a number of years. However, a consortium led by two California-based companies, Affymetrix Inc. of Santa Clara and its partner Molecular Dynamics of Sunnyvale, garnered the lion's share with an award of $\$ 31.5$ million over 5 years. Affymetrix and Molecular Dynamics will match the government contribution.

The competitive awards themselves,

\section{cular dystrophy.}

Diagnostic devices based on a 'DNA-chip' format are also being developed by several other awardees. Combion, Inc., of Redwood City, California, hopes to build on technology invented at the California Institute of Technology, whereby two-dimensional libraries of oligonucleotides are assembled on a solid matrix by sequential synthesis in a manner analogous to ink-jet printing. These so-called Chem-jet arrays will then be used to develop tests for the diagnosis of genetic disorders, to detect microorganisms and to reconstruct the full sequence of previously unanalysed genetic samples.

California-based Hyseq, Inc., of Sunnyvale plans to develop a biochemical 'super chip', based on the company's sequencing-byhybridization technology licensed from the Energy Department's Argonne National Laboratory. With this technology, unknown DNA is sequenced on a super chip that is subdivided into a grid of 16,000 microplates or 'oligochips', each containing up to 16,000 DNA probes linked to the solid support. This set-up allows thousands of samples to be processed in parallel. The goal for Hyseq is to design a device capable of diagnostically sequencing 100 genes at a time.

Rather than invent a new biochemistry like that above, Molecular Tool proposes producing a miniaturized, integrated microfabricated device for DNA typing, adapted from its technology for DNA analysis called genetic bit analysis. The company is already using it in a standard solid-phase, 96-well format to verify the parentage of thoroughbred racehorses, and now hopes, with the help of experts in microfluidics at the Energy Department's Oak Ridge National Laboratory, to produce a miniaturized genotyping device on a glass chip about the size of a dime. Computer-driven voltage generation will be used to control the flow of liquids within the tiny channels etched into the glass, in the hope that, when fully developed, the device will be able to per-

so-called cooperative agreements, are somewhat unique. Neither grant nor contract, all involve some form of cost sharing by industry. And, compared to the 'arms-length relationship' maintained by other government agencies, staff at the standards institute have more of a "stewardship role", says Stuart Davidson, president of Combion Inc., Redwood City, California, providing both technical and business support, if necessary. form a complete genotyping test of up to 1,000 DNA sites of interest at a fraction of the $\$ 100$ it now costs for genotyping only a few dozen sites.

Two companies - Bruker Analytical Systems, Inc., of Billerica, Massachusetts and GeneTrace Systems, Inc., of Menlo Park, California - both plan to demonstrate the feasibility of using a benchtop DNA sequencing and sizing device to perform routine diagnostic applications. To do this, the companies are attempting to couple - in an automated fashion - proprietary frontend chemistry with time-of-flight mass spectrometry. In contrast to simple hybridization-based methods, having the complete DNA sequence may be necessary for a full diagnostic analysis such as in the screening of cancer-related genetic mutations and to avoid false negatives.

GeneTrace's aim is to produce an instrument capable of sequencing 100 times faster than current systems with a throughput of $1,500,000$ bases per day. The instrument could be a bit pricey, somewhere in the order of $\$ 150,000$. The only way to make that economically feasible, Christopher H. Becker, president and CEO of GeneTrace says, is to have a high enough sample throughput and make the tests inexpensive enough to be widely adopted.

A laser manufacturer from San Jose, California, and the Applied Biosystems division of Perkin-Elmer Corporation plan jointly to develop a more compact, efficient and costeffective blue wavelength, solid-state laser for the next-generation of desktop DNA sequencing systems. One of the limiting factors has been the bulkiness, high cost and inefficiency of the air-cooled argon lasers now in use, says Gordon Mitchard, marketing manager at Uniphase. The company's proposed BioLaser is expected to be at least 100 times more efficient.

D.G.

Many of the projects are ambitious with considerable technical obstacles still to overcome. But the race is now on to see which companies manage to corner the largest share of the DNA-based diagnostics market, which, according to Fortune magazine, could be a \$7-billiona-year industry by the year 2,000. As Molecular Tool's Knapp puts it: "In a sense, the starting gun has now gone off in this area."

DIANE GERSHON 